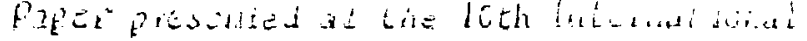

Free Electron Laser Conference,

ierusalem, Isiat - August -9-septomher 2, 1989

\title{
AMPLIFIED SPONTANEOUS EMISSION IN A SINGLE PASS FREE ELECTRON LASER*
}

Li-Hua Yu and Samuel Krinsky

National Synchrotron Light Source

Brookhaven National Laboratory

Upton, New York 11973

BNL --42192

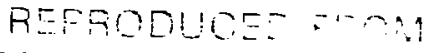

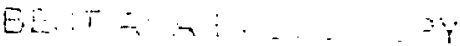

November 1988

DE89 007048

\section{Abstract}

We discuss the relationship of the effective start-up noise in a

single pass free electron laser to the spontaneous radiation emitted in the initial gain length of the wiggler magnet. Also, it is noted that the number of modes in the output is related to the phase space volume occupied by the spontaneous radiation emitted in the first gain length.

\section{DISCLAIMER}

This report was prepared as an account of work sponsored by an agency of the Lnited States Government. Neither the United States Government nur any agency thereof, nor any of their employees, makes any warranty, express $\mathrm{c}$ i implied, or assumes any legal liability or responsibility for the accuracy. comple:eness, or usefulness of any information, apparatus, product, or process disclosed, or represents that its use would not infringe privately owned rights. Reference herein to any specific commercial product. process, or service by trade name, trademark, manufacturer, or otherwise does not necessarily constitute or imply its endorsement, recommendation, or favoring by the United States Government or any agency thereof. The views and opinions of authors expressed herein de not necessarily state or reflect those of the United States Government or any agescy theresf.

*This work was performed under the auspices of the U.S. Department of Energy.

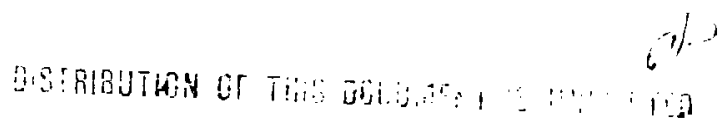


1. Start-Up Noise in One Mode Expressed in Terms of Spontaneous Radiation

The initial value problem determining the start-up of a single pass free electron laser from the Schottky noise in the electron beam has been discussed in Refs. [1-6]. In this note we extend previous work by expressing the output power spectrum of the amplified spontaneous radiation in terms of the spontaneous radiation emitted by the electrons while passing through the first gain length (e-folding length) of the wiggler magnet. In the high-gain regime before saturation, the output power spectrum $\mathrm{dP} / \mathrm{d} \omega$ can be expressed in terms of Moore's [7] exponentially growing guided modes, and one can write $[5,6]$ :

$$
\frac{d P}{d \omega}=\sum_{n, \ell} G_{n \ell}(\omega, z)\left[\left(\frac{d P}{d \omega}\right)_{\text {INPUT }}+\left(\frac{d P_{n \ell}}{d \omega}\right)_{\text {NOISE }}\right] .
$$

Each term in the sum over $n, \ell$ corresponds to a guided mode, or a pair of guided modes. The cross terms $n \neq \ell$ are, in general, small and can be neglected. The contribution from an external coherent EM-wave incident at $z=0$ is $\left(\mathrm{dP}_{\mathrm{n} \ell} / \mathrm{d} \omega\right)$ INPUT, and the contribution from the Schottky noise in the electron beam is $\left(\mathrm{dP}_{\mathrm{n} \ell^{\prime}} / \mathrm{d} \omega\right)$ NOISE. The gain in length $z$ of the free electron laser is described by $G_{n \ell}(\omega, z)$.

In order to obtain simple results with a clear physical $i_{1}$ terpretation, let us restrict our attention to radiation at the resonant wavelength

$$
\lambda_{0}=\frac{\lambda_{h}}{z \gamma_{0}^{2}}\left(1+K^{2}\right) \text {, }
$$

where $\lambda_{W}$ is the period length of the static wiggler magnetic field, $K$ is the dimensionless magnetic field strength parameter [8], and $\gamma_{0}=E_{0} / m^{2}$ is the electron energy in units of the rest nass. The spontaneous power radiated in the forward direction per unit frequency per unit solid angle is [8] (mks units):

$$
\left(\frac{d P}{d w d \Omega}\right)_{N}^{S P O N T}=\frac{2 N^{2} e^{2} \gamma_{0}^{2} K^{2}}{c\left(1+t^{2}\right)^{2} 4 \pi \epsilon_{0}} \frac{I}{e}
$$


where $I$ is the electron beam current and $N$ is the number of wiggler periods traversed. The spontaneous radiation emitted in the first part of the wiggler interacts with the electron beam causing it to bunch, and the bunched electron beam amplifies the radiation. The output power spəctrum of the amplified spontaneous rasiaticn in the fundamental guided mode $n=1$, at the resonant Erequency, can be written

$$
\frac{\mathrm{dP}_{11}}{\mathrm{~d} \omega}=\frac{1}{9} \mathrm{e}^{-2 z / \mathrm{L}}\left(\frac{\mathrm{dP}_{11}}{\mathrm{~d} \omega}\right)_{\text {NOISE }} .
$$

where $L$ is the gain length for the fundamental mode.

One-dimensional theory [2,3] yields the following result for the effective noise power spectrum [5]:

$$
\left(\frac{d P}{d \omega}\right)_{\text {NOISE }}^{l D}=\rho E_{o} / 2 \pi \text {. }
$$

where $\rho$ is the Pierce parameter [9]. For a cylindrical electron beam with circular step-function profile of radius $r_{0}$.

$$
(2 \rho)^{3}=\frac{2 e Z_{o} I}{\pi \gamma_{o} m c^{2}} \frac{1}{2 k_{o} k_{w^{2}}{ }^{2}} \frac{K^{2}}{1+K^{2}},
$$

with $k_{0}-2 \pi / \lambda_{0}, k_{w}=2 \pi / \lambda_{w}$ and $z_{o}=1 / \epsilon_{0} c$. Let us note that the effective noise power spectrum as predicted from one-dimensional theory. Eq. (5), can be rewritten in cerms of the spectrum of the spontaneous radiation emitted in the first gain length of the wiggler. One easily shows:

$$
\left(\frac{d P}{d \omega}\right)_{\text {NOISE }}^{1 D}-\frac{3}{4} \frac{\lambda_{o}^{2}}{\pi r_{o}^{2}}\left(\frac{d P}{d \omega d \Omega}\right)_{N=L}^{S P / \lambda_{\omega}}
$$

where $L^{1 D}$ is the gain length predicted by one-dimensional theory, $\lambda_{W} / L^{1 D}=$ $2 \pi \rho \sqrt{3}$. The right-hand side of Eq. (7) is $3 / 4$ times the power spectrum of the spontaneous radiation emitted into the diffraction solid angle $\Delta \Omega=\lambda_{0}^{2} / \pi r_{0}^{2}$, in the first gain length.

When the electron beam radius $r_{0}$ is large enough, the one-dimensional result of Eq. (5) is a good approximation. However, as the electron beam 
radius $r_{0}$ is reduced, with current I fixed, eventually the one-dimensional approximation breaks down. This is clearly seen by noting that as $r_{0} \rightarrow 0$, I fixed, the Pierce parameter $\rho-r_{0}{ }^{-2 / 3}$, so the effective power spectrum of Eq.

(5) diverges, which is unphysical.

Let us define the diffraction ingle

$$
\theta_{D}=\lambda_{0} / \pi r_{0} \text {, }
$$

and the angle of emission of the spontaneous radiation

$$
\theta_{w}=\sqrt{\lambda_{o} / L} \text {. }
$$

When the radius of the electron beam is large, $\theta_{D} \ll \theta_{W}$, and only a small fraction of the spontaneous radiation feeds into the fundamental mode. In this case, $\left(\theta_{D} \ll \theta_{w}\right)$

$$
\left(\frac{\mathrm{dP}_{11}}{\mathrm{~d} \omega}\right)_{\text {NOISE }} \approx\left(\frac{\mathrm{dP}}{\mathrm{d} \omega}\right)_{\text {NOISE }}^{1 \mathrm{D}}=\frac{3}{4} \pi \theta_{\mathrm{D}}^{2}\left(\frac{\mathrm{dP}}{\mathrm{d} \omega \mathrm{d} \Omega}\right)_{\mathrm{N}=\mathrm{L} / \lambda_{\mathrm{w}}}^{\mathrm{SPONT}} .
$$

The total number of the modes which are excited is on the order of $\left(\theta_{W} / \theta_{D}\right)^{2}$. Now, as the radius of the electron beam is reduced, current held fixed, eventually one reaches the regime $\theta_{w} \ll \theta_{D}$. Now, since the emission angle of the spontaneous radiation is small compared to the diffraction angle, all of the spontaneous power at the resonant frequency should feed into the fundamental mode. The effective noise power spectrum can be computed from Eq. (5.15) of Ref. [6], see Appendix, and one obtains $\left(\theta_{W} \ll \theta_{D}\right)$ :

$$
\left(\frac{\mathrm{dP}_{11}}{\mathrm{~d} \omega}\right)_{\text {NOISE }} \approx \frac{9}{8} \pi \theta_{\mathrm{w}}^{2}\left(\frac{\mathrm{dP}}{\mathrm{d} \omega \mathrm{d} \Omega}\right)_{\mathrm{N}=\mathrm{L} / \lambda_{\omega}}^{\mathrm{SPONT}},
$$

where $L$ is the gain length of the fundamental mode for small radius $r_{0}$. The right-hand side is approximately equal to the total spontaneous power spectrum at the resonant frequency, radiated in the first gain length.

For arbitrary electron bean radius, the effective noise power spectrun, coupling to the fundamental mode, can be determined from Eq. (5.15) of Ref. [6]. The result can be written in the form: 


$$
\left(\frac{d P}{d \omega}\right)_{N O I S E}-F \pi \theta^{2}\left(\frac{d P}{d \omega d \Omega}\right)_{N=L / \lambda}^{S P O N T} .
$$

where $L$ is the gain length corresponding to the given electron beam parameters, and the angle $\theta$ is defined by

$$
\frac{9}{\theta^{2}}=\frac{8}{\theta_{\mathrm{W}}{ }^{2}}+\frac{12}{\theta_{\mathrm{D}}^{2}}
$$

This definition of $\theta$ assures that for large electron beam size $\theta \approx \sqrt{3 / 4} \theta_{D}$, and for small electron beam size $\theta \approx \sqrt{9 / 8} \sigma_{w}$, hence, the form factor $F=F\left(\theta_{w} / \theta_{D}\right)$ satisfies $F(0)=F(\infty)=1$. One finds that $F$ only varies in the range $0.65 \leq F$ $\leq 1$. Further details of the calculation will be given elsewhere.

\section{Number of Transverse Modes in the Output Radiation}

Optical guiding in free electron lasers has been studied by many authors $[7,10,11,12]$. Our work [6] has been based upon the approach introduced by Moore [7], who expanded an input coherent radiation field into a superposition of transverse modes, each evolving exponentially along the axis of the wiggler magnet, with invariant transverse profile. Since different modes have different growth rates, the weights of the different modes vary as the EM-wave propagates through the laser, and hence the transverse profile of the radiation beam also changes. If the radius of the electron beam is not too large, the fundamental mode has the largest growth rate, and hence eventually becomes dominant. In this case, the EM-wave evolves into a single transverse mode, the beam profile becomes constant and this is optical guiding.

It is useful to introduce [6] a dimensionless scaled electron beam radius ã defined up to a multiplicative constant by $\bar{a}^{2} \propto k_{0} x_{0}^{2} / L^{1 D}$, the ratio of the Rayleigh range to the one-dimensional gain length. To be specific, we define

$$
\tilde{a}^{2}=2 \rho\left(2 k_{o} k_{w}\right) r_{0}^{2}
$$


Suppose the current density is held constant while the electron beam radius is increased. When the radius becomes large enough, the gain becomes independent of beam size, and is that given by one-dimensional theory. The parameter ã characterizes the competition between diffraction and gain. When $\bar{a} \gg 1$, the Rayleigh range is much larger than the gain length and diffraction is negligible. In this case, the gain is that given by one-dimensional theory, and many modes are degenerate having the same growth rate. On the other hand, when $\bar{a} \leq 1$, the Rayleigh range becomes smaller than the gain length, and diffraction reduces the growth rate below that predicted by one-dimensional theory. In this case, a single mode has the largest gain, and hence the output of a long wiggler will be dominated by this mode.

To treat the start-up from Schottky noise, it is necessary to describe how the radiation evolves from a source lacalized at a point (electron) into a wave packet. This wave packet is the Green's function of the initial value problem. In our previous work [6] we have shown how to expand the Green's function in terms of Moore's modes. Once the Green's function is known, the contribution frcm all the electrons is determined by carrying out an average over the stochastic ensemble describing the Schottky noise in the electron beam. In this way, the output power is computed, and one finds the frequency spectrum as given in Eq. (5.15) of Ref. [6]. From this one can derive the expressions for the gain and start-up noise presented in Section 1.

In the previous section, we have discussed the relationship of the effective start-up noise to the spontaneous radiation emitted in the first gain length of the wiggler. Now we shall consider the number of transverse modes fed by the spontaneous radiation. We begin by considering $\tilde{a}>1$. In this case, we note that an individual electron passing through a wiggler of length $L^{1 D}$ emits radiation at the resonant frequency into a cone characterized by 
angle [Eq. (9)] $\theta_{\mathrm{w}} \approx{\sqrt{\lambda_{\mathrm{o}} / \mathrm{L}}}^{1 D}$. The phase space volume occupied by the spontaneously emitted radiation from all the electrons is roughly $\left(r_{0} \theta_{w}\right)^{2}$. Since each transverse mode occupies roughly a phase space volume of $\lambda_{0}^{2}$, one sees that the number of modes in the spontaneous radiation field emitted in the initial gain length is approximately

$$
\frac{\left(r_{O} \theta_{w}\right)^{2}}{\lambda_{0}^{2}} \propto \bar{a}^{2} \text {. }
$$

It turns out that the scaled electron beam size $\overline{\mathbf{a}}$ also satisfies ( $\overline{\mathbf{a}} \gg 1$ ):

$$
\overline{\mathrm{a}} \approx \theta_{\mathrm{W}} / \theta_{\mathrm{D}},
$$

where [Eq. (8)] $\theta_{D}-\lambda_{0} / \pi r_{0}$ is the diffraction angle corresponding to radius $r_{0}$ of the electron beam. Hence, the number of modes in the spontaneous radiation field emitted in length $L^{1 D}$ is roughly $\left(\theta_{w} / \theta_{D}\right)^{2}$.

When $\bar{a} \gg 1$, there are many transverse modes in the output radiation field. There are two reasons for this. First, the spontaneous radiation emitted in the first gain length contains many modes, and second, when $\vec{a} \gg 1$, many modes have growth rate degenerate with the fundamental mode. The effective noise power spectrum for one mode is given by Eq. (10), and is approximately equal to the spontaneous power spectrum radiated into the diffraction solid angle $\Delta \Omega=\pi \theta^{2}$ in the initial gain length of the wiggler. We expect that the number of modes in the output is roughly $\left(\theta_{\omega} / \theta_{D}\right)^{2}$. When $\bar{a} \approx 1$, we see from Eq. (15) that the spontaneous radiation emitted in the first gain length occupies a phase space area of $\lambda_{0}^{2}$, hence is transversely coherent and will feed only one mode of the output. The diffraction and emission angles are about equal, $\theta_{D} \approx \theta_{w}$, and all of the spontaneous radiation feeds into one mode. The gain is still close to that given by one-dimensional theory, and this is the case considered by $\mathrm{Kim}$ [5]. If one now considers $\tilde{a} \ll 1$, because of diffraction the phase space volume remains at $\lambda_{0}^{2}$, and only a single mode is excited. The emission angle becomes small compared to the diffraction angle, $\theta_{\mathbf{w}} \ll \theta_{D}$, 
and 211 the radiation feeds into one mode, but Eq. (10) is in this case an over-estimate of the effective noise power spectrum. The proper result is given in Eq. (11), and we see that the effective noise power spectrun is approximately equal to the total spontaneously radiated power spectrum emitted in the first gain length of the wiggler. 


\section{References}

[1] H.A. Haus, IEEE J. Quant. Electron. QE-17, 1427 (1981)

[2] J.M. Wang and L.H. Yu, Nucl. Instrum. Methods A250, 484 (1986).

[3] K.J. Kim, Nucl. Instrum. Methods A250, 396 (1986).

[4] S. Krinsky, AIP Conf. Proc. 153, 1015 (1987).

[5] K.J. Kim, Phys. Rev. Lett. 57, 1871 (1986).

[6] S. Krinsky and L.H. Yu, Phys. Rev. 35, 3406 (1987).

[7] G.T. Moore, Nucl. Instrum. Methods A239, 19 (1985); Nuc1. Instrum. Methods A250, 381 (1986).

[8] B.M. Kincaid, J. Appl. Phys. 48, 2684 (1977).

[9] R. Bonifacio, C. Pellegrini and L.M. Narducci, Opt. Commun. 50, 373 (1984).

[10] E.T. Scharlemanr, A.M. Sessler and J.S. Wurtele, Phys. Rev. Lett. 54, 1925 (1985).

[11] P. Sprangle and C.M. Tang, Appl. Pkys. Lett. 39, 677 (1981).

[12] N.M. Kro11, P.L. Morton and M.N. Rosenbluth, IEEE J. Quantum Electron. QE-17, 1436 (1981). 


\section{Appendix}

From Eq. (5.15) of Ref. $\{6\}$, one finds

$$
\left(\frac{\mathrm{dP}_{11}}{\mathrm{~d} \omega}\right)_{\text {NOISE }}=\frac{\rho \mathrm{E}_{\mathrm{O}}}{2 \pi}\left|\frac{2 \rho}{\Omega}\right|^{2} \frac{9}{\left|1-\mathrm{F}_{1}(0)\right|^{2}} \int \mathrm{d}^{2} \mathrm{x}\left|\psi_{1}\right|^{2} \int \mathrm{d}^{2} \mathrm{x} u(\mathrm{x})\left|\psi_{1}\right|^{2} \text {. }
$$

We consider only radiation at the resonant frequency, so the detuning $q_{1}=0$. [Note $\left.\mathrm{dq}_{||}=\mathrm{d} \omega / \omega_{\mathrm{o}}\right]$. The coherent frequency shift $\Omega$ is related to the gain length $L$ by $\operatorname{Im} \Omega=1 / k_{w} L$. The transverse profile of the electron beam is specified by $u(x)$, and the transverse profile of the fundanental mode by $\psi_{1}(x)$, with the normalizations $u(0)=1$ and $\int \mathrm{d}^{2} \mathrm{x} \psi_{1}^{2}=1$. The quantity $F_{1}\left(q_{\mid l}\right)$ is defined in Eq. (4.15) of Ref. [6]. Finally, the dimensionless transverse coordinates $\vec{x}$ are related to the dimensioned transverse coordinates $\vec{r}$ by $\vec{x}=\sqrt{2 k_{0} k_{w}} \vec{r}$. 